University of Nebraska - Lincoln

DigitalCommons@University of Nebraska - Lincoln

Kirill Belashchenko Publications

Research Papers in Physics and Astronomy

2011

\title{
Ce-doped EuO: Magnetic properties and the indirect band gap
}

\author{
Pan Liu \\ University of Wyoming \\ Jinke Tang \\ University of Wyoming, jtang2@uwyo.edu \\ Juan A. Colon Santana \\ University of Nebraska-Lincoln \\ Kirill D. Belashchenko \\ University of Nebraska-Lincoln, belashchenko@unl.edu \\ Peter A. Dowben \\ University of Nebraska-Lincoln, pdowben@unl.edu
}

Follow this and additional works at: https://digitalcommons.unl.edu/physicsbelashchenko

Liu, Pan; Tang, Jinke; Colon Santana, Juan A.; Belashchenko, Kirill D.; and Dowben, Peter A., "Ce-doped EuO: Magnetic properties and the indirect band gap" (2011). Kirill Belashchenko Publications. 8.

https://digitalcommons.unl.edu/physicsbelashchenko/8

This Article is brought to you for free and open access by the Research Papers in Physics and Astronomy at DigitalCommons@University of Nebraska - Lincoln. It has been accepted for inclusion in Kirill Belashchenko Publications by an authorized administrator of DigitalCommons@University of Nebraska - Lincoln. 


\title{
Ce-doped EuO: Magnetic properties and the indirect band gap
}

\author{
Pan Liu, ${ }^{1}$ Jinke Tang, ${ }^{1, a)}$ Juan A. Colón Santana,${ }^{2}$ Kirill D. Belashchenko, ${ }^{2}$ \\ and Peter A. Dowben ${ }^{2}$ \\ ${ }^{1}$ Department of Physics and Astronomy, University of Wyoming, Laramie, Wyoming 82071, USA \\ ${ }^{2}$ Department of Physics and Astronomy and the Nebraska Center for Materials and Nanoscience, \\ University of Nebraska-Lincoln, Lincoln, Nebraska 68588-0299, USA
}

(Presented 17 November 2010; received 7 October 2010; accepted 24 October 2010; published online 25 March 2011)

\begin{abstract}
We have prepared and investigated thin films of EuO doped with the rare-earth element cerium. $\mathrm{X}$-ray diffraction, scanning electron microscopy, and energy dispersive X-ray spectroscopy were used to determine the quality of these films prepared by pulsed laser deposition. Ce doping leads to an enhanced Curie temperature near $150 \mathrm{~K}$, close to that seen for oxygen-deficient $\mathrm{EuO}_{1-x}$. However, the magnetization of $\mathrm{Ce}$-doped $\mathrm{EuO}$ exhibits differences from that observed for Gd-doped and oxygen-deficient samples. The high-resolution angular-resolved photoemission from Ce-doped EuO reveals filling of conduction-band states near the $\mathrm{X}$ point. This indicates that the band gap in EuO is indirect, and that at $2 \%$ doping Ce-doped $\mathrm{EuO}_{1-x}$ is at least semimetallic. (C) 2011 American Institute of Physics. [doi:10.1063/1.3544478]
\end{abstract}

Europium monoxide $(\mathrm{EuO})$ is a well-known ferromagnetic semiconductor. Stoichiometric EuO has a Curie temperature $\left(T_{C}\right)$ of $69 \mathrm{~K}$ and a band gap of $\sim 1.12 \mathrm{eV} .{ }^{1,2}$ There are some spectacular phenomena for this material with electron doping, such as a metal-to-insulator transition and colossal magnetoresistance, where the resistivity change can exceed 810 orders of magnitude. ${ }^{3,4}$ The Curie temperature can also be enhanced significantly by electron doping via rare-earth atoms $^{5-7}$ or oxygen vacancies. ${ }^{6-9}$ Recent studies have shown that the conduction band of EuO is spin split by $\sim 0.6 \mathrm{eV}$ in its ferromagnetic state, which leads to an almost $100 \%$ spin polarization of the electrons close to the conduction band. ${ }^{10}$

Epitaxial or strongly textured EuO (100) films can be grown on a Si (100) wafer with high-quality interface by reactive thermal evaporation of Eu or molecular beam epitaxy. ${ }^{11}$ Our previous study demonstrates that high-quality EuO films can be grown using pulsed laser deposition (PLD) with metal targets. ${ }^{8}$ Both stoichiometric and oxygen-deficient europium oxide films can be prepared by controlling the annealing conditions of the Si wafers.

In this paper, we have succeeded in preparing EuO, $\mathrm{EuO}_{1-x}$, and $\mathrm{Eu}_{0.98} \mathrm{Ce}_{0.02} \mathrm{O}_{1-x}$ films on $\mathrm{Si}$ (100) wafers via PLD. X-ray diffraction (XRD) and scanning electron microscopy-energy dispersive $\mathrm{X}$-ray spectroscopy (SEM-EDX) were used to investigate the films and showed they have the fcc rock salt crystal structure with and without the doping of the rare-earth element cerium $(\mathrm{Ce})$. Ce doping changes the lattice constant and leads to the enhancement of the Curie temperature to $150 \mathrm{~K}$, close to that of oxygen-deficient $\mathrm{EuO}_{1-x}{ }^{8}$ There are differences in the magnetization curves that suggest that the effects of Ce doping differ from that caused by oxygen vacancies.

The EuO films and Ce-doped EuO films were prepared using PLD in a vacuum of $10^{-5}$ Torr with flowing $\mathrm{H}_{2}$ at

\footnotetext{
${ }^{\text {a) }}$ Author to whom correspondence should be addressed. Electronic mail: jtang2@uwyo.edu.
}

room temperature. Before the deposition, the $\mathrm{Si}$ wafers were annealed at $750{ }^{\circ} \mathrm{C}$ under pure $\mathrm{H}_{2}$ gas in order to remove the native $\mathrm{SiO}_{2}$ surface layer from the wafers. Annealing times of 30 and $50 \mathrm{~min}$ resulted in stoichiometric and oxygen-deficient films, respectively. The PLD targets were either an Eu (99.9\%) metal or a mixture of $\mathrm{Eu}(99.9 \%)$ and $\mathrm{Ce}(99.9 \%)$ metals, and the purity of the $\mathrm{H}_{2}$ gas used during the deposition is $99.995 \%$. The composition of the grown Ce-doped $\mathrm{EuO}$ was confirmed by EDS to be $\mathrm{Eu}_{0.98} \mathrm{Ce}_{0.02} \mathrm{O}_{1-x}$. XRD data were collected using a Philips X'Pert diffractometer using $\mathrm{Cu} K \alpha$ radiation. The combined photoemission and inverse photoemission studies were carried out as described elsewhere, ${ }^{12}$ while angle-resolved high resolution photoemission studies were used to map the wave-vector-dependent density of states in the vicinity of the Fermi level, following a recipe also described elsewhere. ${ }^{13}$

As shown in Fig. 1, the XRD pattern of $\mathrm{Eu}_{0.98} \mathrm{Ce}_{0.02} \mathrm{O}_{1-x}$ is consistent with a film having the fcc rock salt crystal

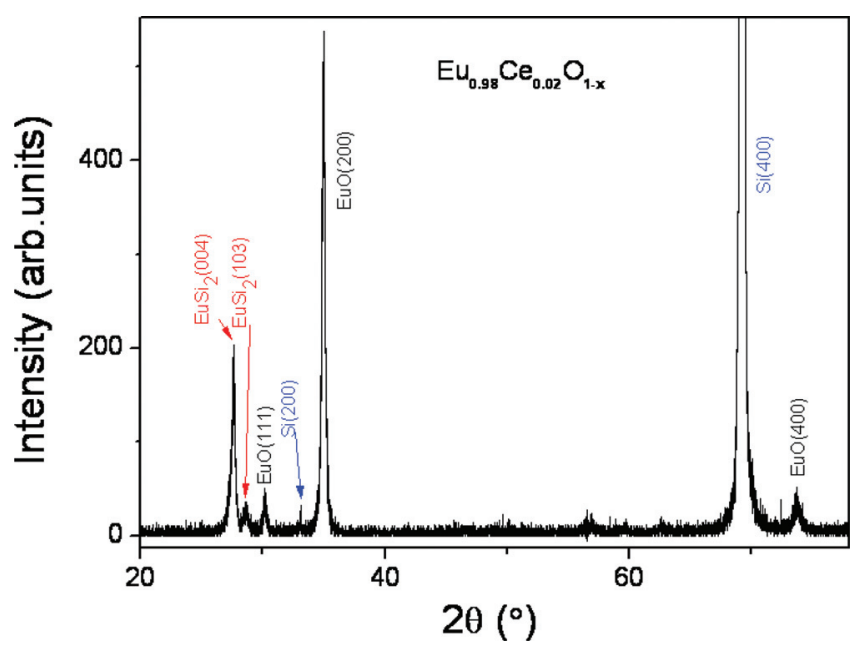

FIG. 1. (Color online) XRD patterns of $\mathrm{Eu}_{0.98} \mathrm{Ce}_{0.02} \mathrm{O}_{1-x}$ film. 
TABLE I. Comparison of lattice constants and Curie temperatures for $\mathrm{EuO}, \mathrm{EuO}_{1-x}, \mathrm{Eu}_{0.96} \mathrm{Gd}_{0.04} \mathrm{O},{ }^{\mathrm{a}}$ $\mathrm{Eu}_{0.96} \mathrm{Gd}_{0.04} \mathrm{O}_{1-x},{ }^{\text {a }}$ and $\mathrm{Eu}_{0.98} \mathrm{Ce}_{0.02} \mathrm{O}_{1-x}$.

\begin{tabular}{lccccc}
\hline \hline & $\mathrm{EuO}$ & $\mathrm{EuO}_{1-x}$ & $\mathrm{Eu}_{0.96} \mathrm{Gd}_{0.04} \mathrm{O}$ & $\mathrm{Eu}_{0.96} \mathrm{Gd}_{0.04} \mathrm{O}_{1-x}$ & $\mathrm{Eu}_{0.98} \mathrm{Ce}_{0.02} \mathrm{O}_{1-x}$ \\
\hline Dominant orientation & $\langle 200\rangle$ & $\langle 200\rangle$ & $\langle 111\rangle$ & $\langle 200\rangle$ & $\langle 200\rangle$ \\
Lattice constant (nm) & 0.5131 & 0.5106 & 0.5118 & 0.5091 & 0.5105 \\
Curie temperature (K) & 70 & 150 & 120 & 145 & 150 \\
\hline \hline
\end{tabular}

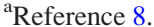

structure expected of EuO. It shows that the stacking planes are mostly aligned with the $\langle 200\rangle$ orientation as reported from prior works. ${ }^{5,11}$ The XRD provides a good indication that the Ce-doped $\mathrm{EuO}$ film is of high quality and strongly textured. There is evidence of silicide formation as peaks of $\mathrm{EuSi}_{2}$ were observed in the film.

Table I compares the lattice constant and Curie temperature of five different samples we have investigated. The lattice constants $a$ determined from the (200) peaks for $\mathrm{Eu}_{0.98} \mathrm{Ce}_{0.02} \mathrm{O}_{1-x}$ are consistent with the presence of $\mathrm{Ce}$. While $a=0.5131 \mathrm{~nm}$ for $\mathrm{EuO}$, this value decreases to $0.5105 \mathrm{~nm}$ for $\mathrm{Eu}_{0.98} \mathrm{Ce}_{0.02} \mathrm{O}_{1-x}$ because of the smaller $\mathrm{Ce}^{3+}$ radius and oxygen deficiency. Because the doping level for Ce used here is a little lower than the Gd doping level, and because the radius of $\mathrm{Ce}^{3+}$ is a little larger than that of $\mathrm{Gd}^{3+}$, the lattice constant of Ce-doped $\mathrm{EuO}_{1-x}$ is larger than that of Gd-doped $\mathrm{EuO}_{1-x}$.

Figure 2 shows the magnetization versus temperature $M(T)$ curves of $\mathrm{EuO}, \mathrm{EuO}_{1-x}$, and $\mathrm{Eu}_{0.98} \mathrm{Ce}_{0.02} \mathrm{O}_{1-x}$ measured in a field of $H=500 \mathrm{Oe}$. The ferromagnetic transition of the stoichiometric $\mathrm{EuO}$ is $\sim 70 \mathrm{~K}$, whereas both $\mathrm{EuO}_{1-x}$ and $\mathrm{Eu}_{0.98} \mathrm{Ce}_{0.02} \mathrm{O}_{1-x}$ exhibit a very-much-enhanced Curie temperature $\left(T_{C}\right)$ of $150 \mathrm{~K}$. The distinct "double-dome" shape in $M(T)$ and higher $T_{C}$ of $\mathrm{EuO}_{1-x}$ are characteristic of oxygen-deficient EuO. Similar results on $\mathrm{EuO}_{1-x}$ were observed by others, e.g., Borukhovich et al. ${ }^{9}$ Although these two films have similar $T_{C}$, for $\mathrm{Eu}_{0.98} \mathrm{Ce}_{0.02} \mathrm{O}_{1-x}$, the shape of $M(T)$ is different from the double-dome feature one sees with $\mathrm{EuO}_{1-x}$ films prepared by the same methods. For

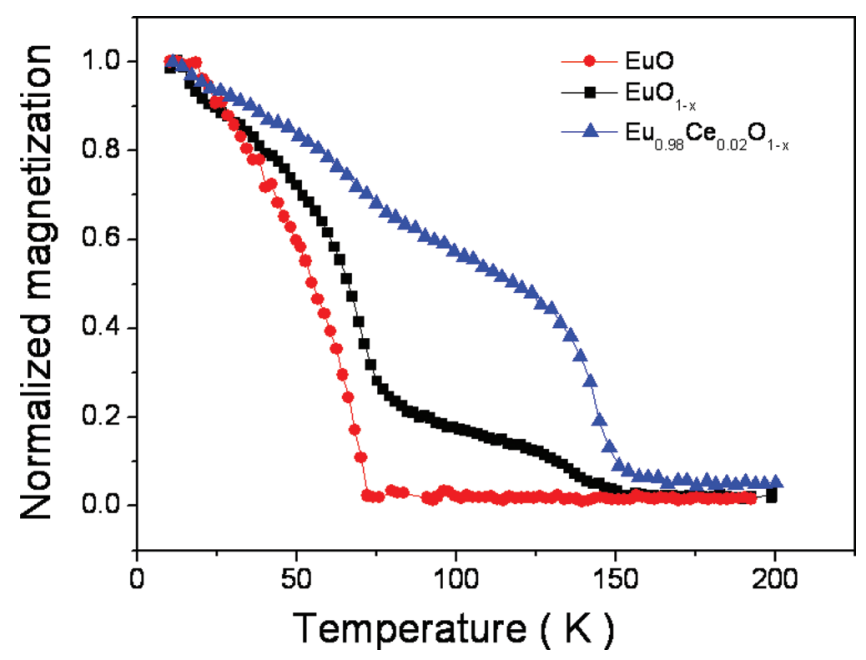

FIG. 2. (Color online) Magnetization as a function of temperature of EuO, $\mathrm{EuO}_{1-x}$, and $\mathrm{Eu}_{0.98} \mathrm{Ce}_{0.02} \mathrm{O}_{1-x}$ films grown by PLD in vacuum and under $\mathrm{H}_{2}$ flow.
$\mathrm{EuO}_{1-x}$, there are two noticeable changes in the magnetization, particularly the differential magnetization (the change in magnetization with temperature), at around 70 and $150 \mathrm{~K}$. For $\mathrm{Eu}_{0.98} \mathrm{Ce}_{0.02} \mathrm{O}_{1-x}$, there is no similarly distinctive drop in magnetization suggestive of a metamagnetic transition (a change from one type of ferromagnetism to another). These differences, between $\mathrm{Eu}_{0.98} \mathrm{Ce}_{0.02} \mathrm{O}_{1-x}$ and $\mathrm{EuO}_{1-x}$ films, can be seen clearly in Fig. 3, in which the derivative of the magnetization $d M / d T$ is shown as a function of temperature. With the $d M / d T$ vs $T$ plot, EuO has one peak at $70 \mathrm{~K}$, which is obviously corresponding to its ferromagnetic transition. Both $\mathrm{EuO}_{1-x}$ and $\mathrm{Eu}_{0.98} \mathrm{Ce}_{0.02} \mathrm{O}_{1-x}$ have two peaks in $d M /$ $d T$, one dominant and the other smaller. The dominant peak occurs at $70 \mathrm{~K}$ and the smaller one at $150 \mathrm{~K}$ for $\mathrm{EuO}_{1-x}$. On the other hand, for $\mathrm{Eu}_{0.98} \mathrm{Ce}_{0.02} \mathrm{O}_{1-x}$, the dominant peak occurs at $150 \mathrm{~K}$ and the peak at $70 \mathrm{~K}$ is rather small. This suggests that most of the magnetic moments are ordered at temperatures as high as $150 \mathrm{~K}$, in contrast to $\mathrm{EuO}_{1-x}$.

It is known that the magnetization of $\mathrm{EuO}$ arises from the $4 f^{7}$ electron configuration of $\mathrm{Eu}^{2+}$. As suggested by Mauger and Godart, ${ }^{1}$ carriers due to oxygen vacancies or doping with rare-earth elements can be excited into the conduction band, which will effectively enhance the magnetic coupling between $\mathrm{Eu}^{2+} 4 f$ local moments via $5 d$ conduction electrons due to the $4 f-5 d$ exchange interaction. This $4 f-5 d$ exchange interaction is responsible for the enhancement of $T_{C}$ beyond $70 \mathrm{~K}$. One expects similar values of the saturation magnetization both below and above $70 \mathrm{~K}$ based on this local moment model. An alternative model, based on the exchange splitting of the conduction electrons, predicts a smaller magnetization due to the on-site polarization of conduction

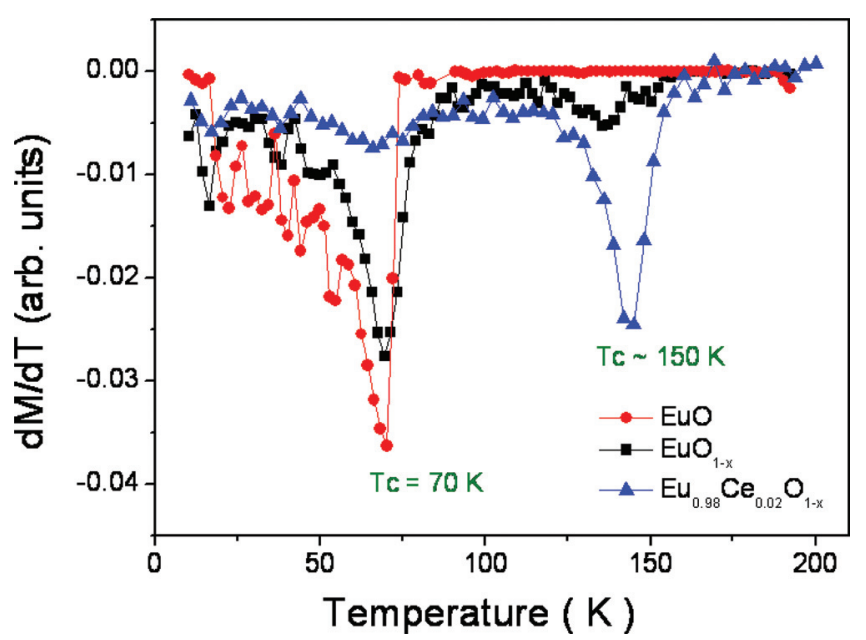

FIG. 3. (Color online) Derivative of the magnetization as a function of temperature of $\mathrm{EuO}, \mathrm{EuO}_{1-x}$, and $\mathrm{Eu}_{0.98} \mathrm{Ce}_{0.02} \mathrm{O}_{1-x}$ films. 


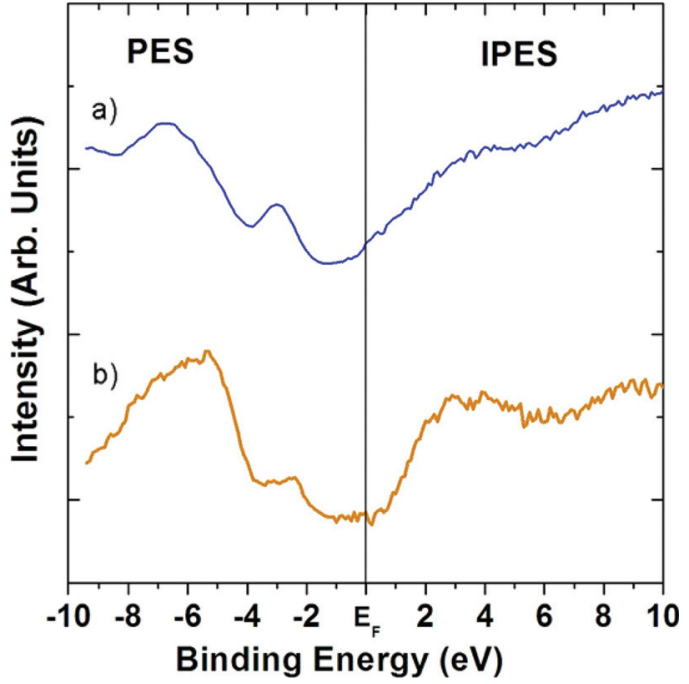

FIG. 4. (Color online) The combined photoemission (left) and inverse photoemission (right) of (a) $\mathrm{EuO}$ (100) and (b) $\mathrm{Eu}_{0.98} \mathrm{Ce}_{0.02} \mathrm{O}_{1-x}$ (100).

electrons between 70 and $150 \mathrm{~K}$ with a much-reduced moment contribution from the $\mathrm{Eu}^{2+}$ moments until the temperature is reduced below $70 \mathrm{~K} .{ }^{14}$

The magnetization curves of $\mathrm{Eu}_{0.98} \mathrm{Ce}_{0.02} \mathrm{O}_{1-x}$ taken at 120 and $50 \mathrm{~K}$ show that saturation magnetization is similar. This behavior is unique to $\mathrm{Ce}$-doped $\mathrm{EuO}$ and was not observed with Gd-doped EuO. ${ }^{8}$ In our study of $\mathrm{EuO}_{1-x}$ and Gd-doped $\mathrm{EuO}$, the saturation magnetization above $70 \mathrm{~K}$ was found to be significantly smaller than below $70 \mathrm{~K} .{ }^{8} \mathrm{Ce}-$ rium is somewhat unique in that because of strong $4 f-5 d$ hybridization, there is an additional $4 f$ local moment contribution to the electronic structure in the vicinity of the Fermi energy. ${ }^{15-18}$ This Ce local moment contribution may aid to keep $\mathrm{Eu}^{2+} 4 f$ local moments still aligned in $\mathrm{EuO}$ at $120 \mathrm{~K}$, but from the data presented here, we can only speculate as the underlying mechanism is not clear.

Although the electronic structures of Ce-doped $\mathrm{EuO}$ and EuO do not appear to differ significantly in the combined photoemission and inverse photoemission studies (Fig. 4), the angle resolution photoemission studies of $\mathrm{Eu}_{0.98} \mathrm{Ce}_{0.02} \mathrm{O}_{1-x}$ film indicate that there is filling of electron pockets, of an otherwise empty conduction band. As shown in Fig. 5, there is filling of electron pockets evident in the wave-vector-dependent photoemission density of states at the Fermi energy. This is not observed in the undoped $\mathrm{EuO}$ films. This filling of the conduction band minimum occurs at the Brillouin zone edge, indicating that the band gap in $\mathrm{EuO}$ is indirect.

In summary, we have prepared the high-quality europium oxide films with $\mathrm{Ce}$ doping on the $\mathrm{Si}(100)$ wafer via PLD. The $T_{C}$ of $\mathrm{Eu}_{0.98} \mathrm{Ce}_{0.02} \mathrm{O}_{1-x}$ film is significantly enhanced by Ce doping and oxygen vacancies to $150 \mathrm{~K}$, similar to $\mathrm{EuO}_{1-x}$, however, the magnetization $M(T)$ and $M(H)$ are remarkably different. Angular-resolved photoemission indicates that the band gap in $\mathrm{EuO}$ is indirect.

We would like to thank Dr. Norbert Swoboda-Colberg for his help with the SEM and EDX measurements. This

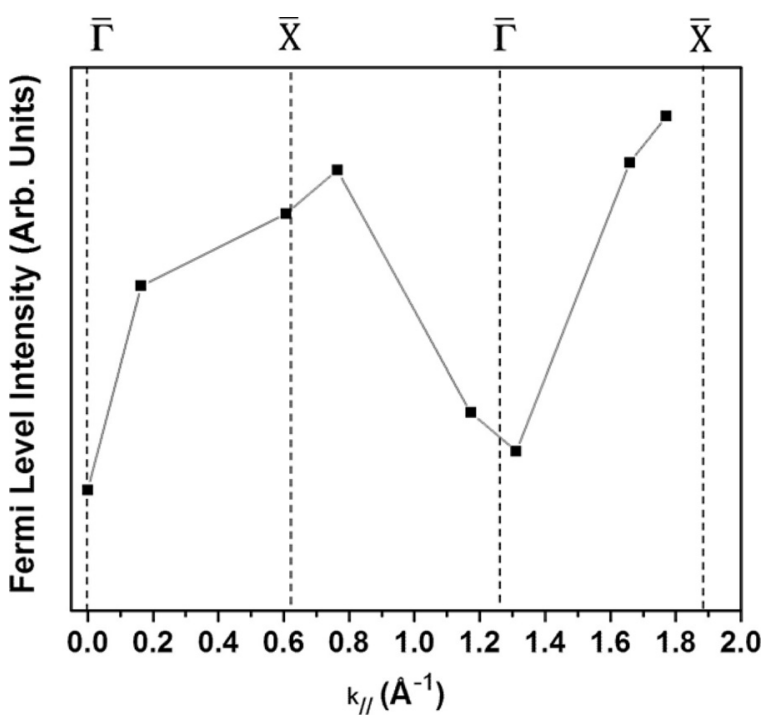

FIG. 5. The angle-resolved photoemission intensities at the Fermi level for $\mathrm{Eu}_{0.98} \mathrm{Ce}_{0.02} \mathrm{O}_{1-x}(100)$, using a photon energy of $25 \mathrm{eV}$. The increase in intensity is indicative of electron pocket formation at the Brillouin zone edge along the $\Gamma$ to $X$ of the surface Brillouin zone.

work was supported by the National Science Foundation (DMR-0852862 and MRSEC DMR-0820521) and the Defense Threat Reduction Agency (Grant No HDTRA1-071-0008).

${ }^{1}$ A. Mauger and C. Godart, Phys. Rep. 141, 51 (1986).

${ }^{2}$ N. Tsuda, K. Nasu, A. Fujimori, and K. Siratori, Electronic Conduction in Oxides, Springer Series in Solid-State Sciences (Springer, New York, 2000).

${ }^{3}$ M. R. Oliver, J. O. Dimmock, A. L. McWhorter, and T. B. Reed, Phys. Rev. B 5, 1078 (1972).

${ }^{4}$ Y. Shapira, S. Foner, R. L. Aggarwal, and T. B. Teed, Phys. Rev. B 8, 2316 (1973).

${ }^{5}$ A. Schmehl, V. Vaithyanathan, A. Herrnberger, S. Thiel, C. Richter, M. Liberati, T. Heeg, M. Röckerath, L. F. Kourkoutis, S. Mühlbauer, P. Böni, D. A. Muller, Y. Barash, J. Schubert, Y. Idzerda, J. Mannhart, and D. G. Schlom, Nature Mater. 6, 882 (2007).

${ }^{6}$ T. Matsumoto, K. Yamaguchi, M. Yuri, K. Kawaguchi, N. Koshizaki, and K. Yamada. J. Phys. Condens. Matter 16, 6017 (2004).

${ }^{7}$ H. Miyazaki, H. J. Im, K. Terashima, S. Yagi, M. Kato, K. Soda, T. Ito, and S. Kimmura, Appl. Phys. Lett. 96, 232503 (2010).

${ }^{8}$ X. Wang, P. Liu, K. A. Fox, J. Tang, J. A. Colón Santana, K. Belashchenko, P. A. Dowben, and Y. Sui, IEEE Trans. Magn. 46, 1879 (2010).

${ }^{9}$ A. S. Borukhovich, V. G. Bamburov, and A. A. Sidorov, J. Magn. Magn. Mater. 73, 1 (1988).

${ }^{10}$ P. G. Steeneken, L. H. Tjeng, I. Elfimov, G. A. Sawatzky, G. Ghiringhelli, N. B. Briikes, and D. J. Huang, Phys. Rev. Lett. 88, 047201 (2002).

${ }^{11}$ J. Lettieri, V. Vaithyanathan, S. K. Eah, J. Stephens, V. Sih, D. D. Awschalom, J. Levy, and D. G. Schlom, Appl. Phys. Lett. 83, 975 (2003).

${ }^{12}$ J. Xiao and P. A. Dowben, J. Mater. Chem. 19, 2172 (2009).

${ }^{13}$ N. Wu, R. F. Sabirianov, W. N. Mei, Y. B. Losovyj, N. Lozova, M. Manno, C. Leighton, and P. A. Dowben, J. Phys. Condens. Matter 21, 295501 (2009).

${ }^{14}$ M. Arnold and J. Kroha, Phys. Rev. Lett. 100, 046404 (2008).

${ }^{15}$ Yu. Kucherenko, S. L. Molodtsov, M. Heber, and C. Laubschat, Phys. Rev. B 66, 155116 (2002).

${ }^{16}$ Yu. S. Dedkov, M. Fonin, Yu. Kucherenko, S. L. Molodtsov, U. Rüdiger, and C. Laubschat, Phys. Rev. B 76, 073104 (2007).

${ }^{17}$ D. M. Wieliczka, C. G. Olson, and D. W. Lynch, Phys. Rev. B 29, 3028 (1984).

${ }^{18}$ D. M. Wieliczka, J. H. Weaver, D. W. Lynch, and C. G. Olson, Phys. Rev. B 26, 7056 (1982). 